\title{
A Comparison between Neuromuscular Effects of Parathion and Paraoxon on Chick Biventer Cervicis Nerve-Muscle and the Reversal of their Effects by Pralidoxime
}

\author{
Gholamreza Poorheidari ${ }^{1,2}$, Alireza Shahriary², and Mahdi Mashhadi Akbar Boojar1,2,* \\ ${ }_{1}^{1}$ Department of Pharmacology and Toxicology, Faculty of Pharmacy, Baqiyatallah University of Medical Sciences, Tehran, Iran \\ ${ }^{2}$ Chemical Injuries Research Center, Baqiyatallah University of Medical Sciences, Tehran, Iran
}

* Corresponding author: Mahdi Mashhadi Akbar Boojar, Department of Pharmacology and Toxicology, Faculty of Pharmacy, Baqiyatallah University of Medical Sciences, Tehran, Iran; Chemical Injuries Research Center, Baqiyatallah University of Medical Sciences, Tehran, Iran. Tel: +982187555414; Email: mahdimashhadi@yahoo.com

Received 2020 October 23; Revised 2020 November 28; Accepted 2021 January 04.

\begin{abstract}
Background: It is generally believed that the anticholinesterase effect is induced by the organophosphate insecticide parathion only through its bioactive metabolite (i.e., paraoxon) that is created in the liver.

Objectives: This study aimed to evaluate the intrinsic anticholinesterase effect of parathion, compared to its main metabolite.

Methods: This study has been conducted to prepare the isolated chick biventer cervicis nerve-muscle using the twitch tension recording method.

Results: According to the results, paraoxon $(0.1 \mu \mathrm{M})$ induced a highly significant increase (more than $100 \%)$ in the twitch height, while higher concentrations $(0.3$ and $1 \mu \mathrm{M})$ induced partial or total contractures. Furthermore, parathion induced almost the same percentage of increase in the twitch height at $1 \mu \mathrm{M}$ and partial or total contractures at 3 and $10 \mu \mathrm{M}$. It should be noted that pralidoxime (2-PAM), at $300 \mu \mathrm{M}$, reversed the effects of paraoxon and its parent (i.e., parathion).

Conclusion: These results demonstrate that both parathion and its metabolite inhibit the acetylcholinesterase enzyme which can be reactivated by pralidoxime, whereas parathion is about 10 times less potent, compared to its metabolite. Therefore, the intrinsic toxic effects of parathion, regardless of its metabolite, should be considered in future studies.

Keywords: Chick biventer cervicis nerve-muscle, Organophosphate, Paraoxon, Parathion, Pralidoxime
\end{abstract}

\section{Background}

Nerve agents are a group of organophosphorus compounds that have been used as military weapons due to their physicochemical properties and toxicity (1). These chemicals have been used in many cases in terrorist attacks and warfare, such as the Halabja chemical attack of the Iran-Iraq War in 1988 (2-4). In general, organophosphorus compounds are most widely used as insecticides and pesticides in agriculture. In addition to the war and terrorist poisonings caused by nerve agents, contamination by organophosphorus compounds is a serious health problem with more than three million poisonings and 200,000 deaths worldwide annually (5).

More than 200 types of organophosphates and 25 types of acetylcholinesterase inhibitor carbamates have been synthesized so far which are accompanied by an increasing number. The organophosphorus insecticides are highly diverse and vary in different properties, such as fat solubility, half-life, active metabolites, antidote bonding, and delayed neuropathy. The criterion for the classification of these compounds is based on the symptoms and severity of poisoning $(6,7)$. Currently, much evidence has evaluated the effects of organophosphorus compounds in animal models (8).

One of the most widely used organophosphorus compounds in animal and laboratory studies is parathion and its active metabolite (i.e., paraoxon) (9). Paraoxon, the same as other organophosphorus compounds, irreversibly inhibits the acetylcholinesterase enzyme and produces muscarinic and nicotine symptoms. The administration of paraoxon in animals has also caused progressive myopathy (10).

It is believed that the anticholinesterase effects induce by the organophosphate insecticide parathion mainly through its bioactive metabolite (i.e., paraoxon) that is generated in the liver $(11,12)$. The direct effects of parathion against neuromuscular function (regardless of active metabolites), comparison of the potency of their neuromuscular effects, and possible effects of pralidoxime on unmetabolized parathion have not been investigated in the literature so far.

\section{Objectives}

This study aimed to investigate the effects of different concentrations of parathion and paraoxon on the function of skeletal muscle and reversal of these effects by pralidoxime. 


\section{Methods}

\subsection{Animals and Chemicals}

This experimental study was conducted on the chick biventer cervicis (CBC) isolated from the chicken neck obtained from the laboratory animal center of Baqiyatallah University of Medical Sciences (BUMS), Tehran, Iran. The CBCs were then utilized for the electrophysiological investigation of neuromuscular transmission. The chicks used in this study were about 4-12 days old, weighed 37-47 g, and were fed and maintained under physiological conditions (with access to water and food ad libitum at $24-28^{\circ} \mathrm{C}$ ). Both parathion and paraoxon were prepared from Sigma America (purity of more than 99\%). Furthermore, the solvents and other reagents were purchased from Merck (Germany) or Sigma Chemical Company (Sigma-Aldrich, USA).

\subsection{Experimental procedure}

As the nerve is stimulated (electrical stimulation), this muscle will respond exactly like the rat diaphragm (Twitch). Moreover, when it is exposed to acetylcholine-like compounds, it produces a strong slow contraction that resembles the frog's abdominal smooth muscle response (13). For CBC nerve-muscle preparation, the CBC muscles and associated nerves were dissected from 4-12day-old chicks killed by exposure to a lethal dose of $\mathrm{CO}_{2}$. Totally, two preparations were mounted in 10 $\mathrm{ml}$ tissue baths containing modified Krebs-Henseleit solution maintained at $32^{\circ} \mathrm{C}$, pH 7.3-7.4, and bubbled with $95 \% \mathrm{O}_{2}+5 \% \mathrm{CO}_{2}$. The modified KrebsHenseleit solution was composed of (mM): $\mathrm{NaCl}$, 118.4; $\mathrm{KH}_{2} \mathrm{PO}_{4}, 1.2$; glucose, 11.1; $\mathrm{NaHCO}_{3}, 25$; $\mathrm{CaCl}_{2}, 2.5 ; \mathrm{MgSO}_{4}, 1.4$ and $\mathrm{KCl}, 4.7$ (14). As described by Khodaei et al. (2004), in twitch tension recording, twitches were evoked by stimulating the motor nerve at $0.1 \mathrm{~Hz}$ with pulses $0.2 \mathrm{msec}$ duration and a voltage greater than that required to produce the maximum response (15).

To detect any changes in postsynaptic sensitivity, responses to submaximal concentrations of acetylcholine (1-2 mM), carbachol (20-40 M), and $\mathrm{KCl}$ (20-40 mM) were recorded (in absence of nerve stimulation) before the addition of toxin and at the end of the experiment. The preparations were washed free of these drugs and allowed 20-30 min to stabilize before the application of toxins. In the absence of toxin, the twitch height or responses to exogenously applied acetylcholine, carbachol, or $\mathrm{KCl}$ did not change in the control experiment groups (up to at least $2 \mathrm{~h}$ ). Twitches and contractures were recorded isometrically using Washington, Grass model 79 and Grass model 79B polygraphs, and SRI or Grass FT03 force transducers (16).

The nerve and muscle were placed at $0.1 \mu \mathrm{M}$ paraoxon concentration solutions, and the impulses were recorded for $60 \mathrm{~min}$. Pralidoxime $300 \mu \mathrm{M}$ concentrations were also used in a separate set of experiments with paraoxon, and impulses were recorded for $60 \mathrm{~min}$.

\subsection{Ethical consideration}

This study was carried out regarding the ethical recommendations of laboratory animal care of BUMS and was approved by the ethics committee and supported by the research deputy of BMSU.

\subsection{Data analysis and presentation}

The data were expressed as a percentage of impulse height, and one-way ANOVA was used to compare the results with those of the control group. All statistical tests were performed in SPSS software (version 20.0), and a p-value less than 0.05 was considered statistically significant. In total, six tissues were included in each test, and all data were expressed as mean \pm SE.

\section{Results}

Figure 1 demonstrates the effects of paraoxon on twitch height in response to electrical stimulation and the reversal of that effect by pralidoxime. Furthermore, paraoxon, at $0.1 \mu \mathrm{M}$, induced a significant increase (more than 100\%) in the twitch height. The maximum contraction was obtained after 20 min of exposure. Moreover, higher concentrations of paraoxon $(0.3$ and $1 \mu \mathrm{M})$ induced partial or total contractures. Pralidoxime, at $300 \mu \mathrm{M}$, reversed (when it was used as post-treatment $20 \mathrm{~min}$ after the exposure to a toxin) the increasing effect of paraoxon in the twitch height.

Similarly, parathion, at $1 \mu \mathrm{M}$, induced almost the same percentage of increase in the twitch height (Figure 2), while higher concentrations $(3,10$, and 30 $\mu \mathrm{M}$ ) induced partial or total contractures, and in some cases, after 45-60 min, the preparation was not responding to the electrical stimulation anymore.

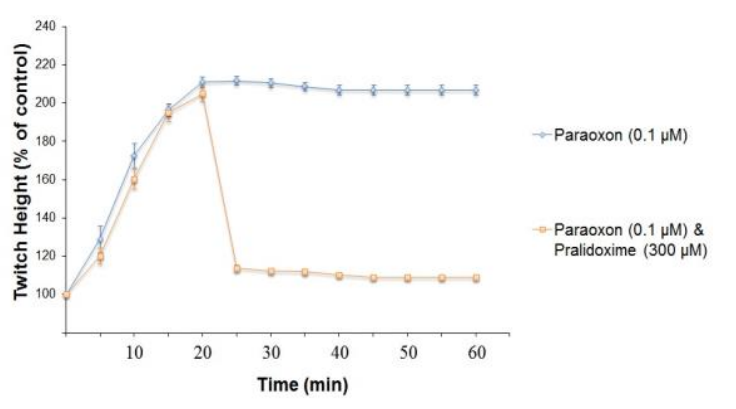

Figure 1. Time-dependent elevation of muscle impulses after electrical neuromuscular stimulation of chick biventer cervicis which presented as twitch height (\% of control) after exposure to paraoxon ( 0.1 micro molar) or parathion ( 0.1 micro molar) and pralidoxime (300 micro molar) at 20 min post-exposure. Each point represents the mean $\pm \mathrm{SE}$ of the six experiments. 


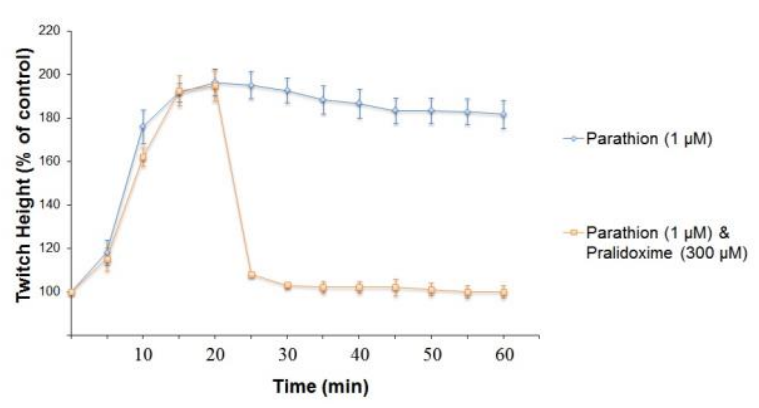

Figure 2. Time-dependent elevation of muscle impulses after electrical neuromuscular stimulation of chick biventer cervicis which presented as twitch height (\% of control) after exposure to parathion (1 micro molar) or parathion ( 0.1 micro molar) and pralidoxime (300 micro molar) at 20 min post-exposure. Each point represents the mean $\pm \mathrm{SE}$ of the six experiments.

However, pralidoxime, at $300 \mu \mathrm{M}$, reversed the twitch height (when it was used as post-treatment $20 \mathrm{~min}$ after the administration of parathion).

\section{Discussion}

One of the toxic effects of nerve agents' warfare and organophosphorus insecticides is skeletal muscle weakness that often occurs in the ocular, neck, bulbar, proximal limb, and respiratory muscles (17, 18). Oximes can prevent or reverse these toxic effects on striated muscles (19). Several studies have been performed to evaluate the enzymatic effects of organophosphate pesticides; however, fewer functional studies have been performed in this regard so far (20-22).

Parathion is an organophosphate insecticide, and it was believed that it acted on the enzyme acetylcholinesterase indirectly (23). After exposure to humans and other organisms, parathion is quickly oxidized to paraoxon, and it is believed that most of the toxic effects of parathion come from this active metabolite (24). However, the functional potency of parathion in comparison to paraoxon was not defined. There are several studies on the different biological aspects of these compounds; however, our investigation was the first in its kind that studied and compared the neuromuscular effects of parathion and paraoxon on $\mathrm{CBC}$ nerve-muscle.

The results of current and recent studies show that muscle responses to the effects of different concentrations of paraoxon and reversal of these effects by pralidoxime effects are significant, repetitive, and reliable. Moreover, this method was used to investigate the cholinergic-nicotinic effects of different substances, and it is considered very useful for the study of striated muscle function $(16,25)$.

However, our preliminary results suggest that parathion itself also has an anticholinesterase activity which has not been reported in the literature. Despite the general belief, parathion, the same as its active metabolite paraoxon, induced a similar increase in response to electrical stimulation of the nerve of the preparation. The results of the present study suggest that parathion does not necessarily need to turn into its metabolite in order to be toxic. These findings were consistent with those observed by other researches in enzymatic studies (26). Reversal of these effects by pralidoxime indicates that observed effects of parathion are also due to the inhibition of acetylcholinesterase enzyme. Although parathion showed to be considerably less potent than paraoxon, it seemed to have some destructive effects on the tissue, which needs more investigations. It has been confirmed that the greatest effect of paraoxon anticholinesterase activity occurs in the striated muscles within $30 \mathrm{~min}$ after exposure, whereas myopathy develops within $24 \mathrm{~h}$ (27).

\subsection{Limitations and suggestions}

Due to the ability of a whole living organism to convert parathion to paraoxon, it is practically impossible to compare the potency of these two compounds with the activity of cholinesterase under in vivo conditions. Moreover, it is not possible to compare the chronic and sub-chronic toxicity of parathion with that of paraoxon. Therefore, it is suggested that future studies compare the biological activities of these two compounds and similar compounds under in vitro conditions.

\section{Conclusion}

These findings indicate that both parathion and its major metabolite (i.e., paraoxon) inhibits the acetylcholinesterase enzyme in striated muscle and leads to an increase in the twitch height which can be deactivated by pralidoxime although parathion is about 10ntimes weaker than paraoxon. Consequently, the intrinsic toxic effects of parathion, regardless of its metabolite, should be considered in future investigations.

\section{Acknowledgments}

The authors thank Dr. Abdol Majid Cheraghali for comments that greatly improved the manuscript.

\section{Footnotes}

Authors' Contribution: Gholamreza Poorheidari developed the theoretical framework and performed the analytic calculations as well as numerical simulations. Alireza Shahriary and Mahdi Mashhadi Akbar Boojar contributed to the final version of the manuscript. Gholamreza Poorheidari supervised the project.

Conflict of Interests: The authors have no conflict of 
interest to declare.

Ethical Approval: This study was carried out regarding the ethical recommendations of laboratory animal care of BUMS and was approved by the Ethics Committee of Research Deputy at BMSU.

Funding/Support: Nil.

Financial Disclosure: None declared.

Informed consent: Nil.

\section{References}

1. Vucinic S, Antonijevic B, Tsatsakis AM, Vassilopoulou L, Docea $\mathrm{AO}$, et al. Environmental exposure to organophosphorus nerve agents. Environ Toxicol Pharmacol. 2017;56:163-71. doi: 10.1016/j.etap.2017.09.004. [PubMed: 28942081].

2. Worek F, Wille T, Koller M, Thiermann H. Toxicology of organophosphorus compounds in view of an increasing terrorist threat. Arch Toxicol. 2016;90(9):2131-45. doi: 10.1007/s00204-016-1772-1. [PubMed: 27349770].

3. Foroutan A. Medical review of Iraqi chemical warfare. Tehran, Iran: Baqiyatallah University of Medical Science; 2002.

4. Foroutan A. A perspective on the medical history of the sacred defense. Tehran, Iran: Sooremehr Publication; 2007.

5. Ai P, Kaiyuan Z, Xinhua L, Changbin L, Buckley NA, Roberts DM. Extracorporeal blood purification for organophosphorus pesticide poisoning. Cochrane Database Syst Rev. 2017; 2017(8):CD006253. doi: 10.1002/14651858.CD006253.pub2. [PubMed: 6483700].

6. Jintana S, Sming K, Krongtong Y, Thanyachai S. Cholinesterase activity, pesticide exposure and health impact in a population exposed to organophosphates. Int Arch Occup Environ Health. 2009;82(7):833-42. doi: 10.1007/s00420-009-0422-9. [PubMed: 19424713].

7. Gupta RC. Classification and uses of organophosphates and carbamates. Toxicology of organophosphate \& carbamate compounds. Massachusetts: Academic Press; 2006. P. 5-24. doi: 10.1016/B978-012088523-7/50003-X.

8. Pereira EF, Aracava Y, DeTolla LJ, Beecham EJ, Basinger GW, Wakayama EJ, et al. Animal models that best reproduce the clinical manifestations of human intoxication with organophosphorus compounds. J Pharmacol Exp Ther. 2014;350(2):313-21. doi: 10.1124/jpet.114.214932. [PubMed: 24907067].

9. Carr RL, Dail MB, Chambers HW, Chambers JE. Species differences in paraoxonase mediated hydrolysis of several organophosphorus insecticide metabolites. $J$ Toxicol. 2015;2015:470189. doi: 10.1155/2015/470189. [PubMed: 25784934].

10. Abdollahi M, Karami-Mohajeri S. A comprehensive review on experimental and clinical findings in intermediate syndrome caused by organophosphate poisoning. Toxicol Appl Pharmacol. 2012;258(3):309-14. doi: 10.1016/j.taap.2011.11.014. [PubMed: 22177963].

11. Mutch E, Williams FM. Diazinon, chlorpyrifos and parathion are metabolised by multiple cytochromes P450 in human liver. Toxicology. 2006;224(1-2):22-32. doi: 10.1016/j.tox.2006.04.024. [PubMed: 16757081].

12. Mutch E, Daly AK, Leathart JB, Blain PG, Williams FM. Do multiple cytochrome $\mathrm{P} 450$ isoforms contribute to parathion metabolism in man? Arch Toxicol. 2003;77(6):313-20. doi: 10.1007/s00204-003-0452-0. [PubMed: 12669189].
13. Rahimi A, Nazemiyeh H, Azarmi Y. Effect of Salvia sahendica extracts on neuromuscular transmission in chick biventer cervicis muscle. Pharm Sci. 2015;21(4):199-204. doi: 10.15171/PS.2015.37.

14. Werner AC, Ferraz MC, Yoshida EH, Tribuiani N, Gautuz JA, Santana MN, et al. The facilitatory effect of Casearia sylvestris Sw.(guaçatonga) fractions on the contractile activity of mammalian and avian neuromuscular apparatus. Curr Pharm Biotechnol. 2015;16(5):468-81. [PubMed: 25751174].

15. Bueno LG, Leite GB, Cruz-Höfling MA, Rodrigues-Simioni L, Oshima-Franco Y. Effects of manganese $(\mathrm{Mn} 2+)$ on neurotoxic and myotoxic activities induced by Crotalus durissus terrificus and Bothrops jararacussu venoms in chick biventer cervicis preparations. J Venom Anim Toxins Incl Trop Dis. 2007; 13(2):479-99.

16. Khodaei N, Shahriary AR, Sahraei H, Noroozzadeh A, Saberi M, Pirzad G, et al. Evaluation of the changes in contractility of Chick biventer cervices nerve-muscle encountered with Paraoxon and Pralidoxime: Introduction of a non-enzymatic method. J Mil Med. 2004;6(1):1-6.

17. Muhammad G, Rashid I, Firyal S. Practical aspects of treatment of organophosphate and carbamate insecticide poisoning in animals. Matrix Sci Pharma. 2017;1(1):10-1.

18. Singh S, Sharma N. Neurological syndromes following organophosphate poisoning. Neurol India. 2000;48(4):308-13. [PubMed: 11146591].

19. Eddleston M, Chowdhury FR. Pharmacological treatment of organophosphorus insecticide poisoning: the old and the (possible) new. Br J Clin Pharmacol. 2016;81(3):462-70. doi: 10.1111/bcp.12784. [PubMed: 26366467].

20. Luo C, Leader H, Radic Z, Maxwell DM, Taylor P, Doctor BP, et al. Two possible orientations of the HI- 6 molecule in the reactivation of organophosphate-inhibited acetylcholinesterase. Biochem Pharmacol. 2003;66(3):387-92. doi: 10.1016/s0006-2952(03) 00237-5. [PubMed: 12907237].

21. Lallement G, Demoncheaux JP, Foquin A, Baubichon D, Galonnier M, Clarençon D, et al. Subchronic administration of pyridostigmine or huperzine to primates: compared efficacy against soman toxicity. Drug Chem Toxicol. 2002;25(3):309-20. doi: 10.1081/dct-120005893. [PubMed: 12173251].

22. Cowan FM, Broomfield CA, Lenz DE, Shih TM. Protective action of the serine protease inhibitor N-tosyl-L-lysine chloromethyl ketone (TLCK) against acute soman poisoning. J App Toxicol. 2001;21(4):293-6. doi: 10.1002/jat.757. [PubMed: 11481662].

23. Eyer F, Meischner V, Kiderlen D, Thiermann H, Worek F, Haberkorn $\mathrm{M}$, et al. Human parathion poisoning. A toxicokinetic analysis. Toxicol Rev. 2003;22(3):143-63. doi: 10.2165/00139709-200322030-00003. [PubMed: 15181664].

24. Isbister GK, Mills K, Friberg LE, Hodge M, O'Connor E, Patel R, et al. Human methyl parathion poisoning. Clin Toxicol. 2007;45(8):956-60. doi: 10.1080/15563650701232745. [PubMed: 17852161].

25. Soukup O, Tobin G, Kumar UK, Binder J, Proska J, Jun D, et al. Interaction of nerve agent antidotes with cholinergic systems. Curr Med Chem. 2010;17(16):1708-18. doi: 10.2174/092986710791111260. [PubMed: 20345348].

26. Jafari M, Pourheidari G. The reactivation effect of pralidoxime in human blood on parathion and paraoxon-induced cholinesterase inhibition. DARU J Pharm Sci. 2006;14(1):37-43.

27. Karalliedde L, Baker D and Marrs TC. Organophosphateinduced intermediate syndrome: aetiology and relationships with myopathy. Toxicol Rev. 2006;25(1):1-14. doi: 10.2165/ 00139709-200625010-00001. [PubMed: 16856766]. 